\title{
Analysis of Varietal Differences in the Fructo-oligosaccharide Accumulation Profile among Onion (Allium cepa L.) Cultivars Grown by Spring-sown Cultivation
}

\author{
Tomoo Maeda ${ }^{1 *}$, Ayumi Watanabe ${ }^{1}$, Daniel Zadrak Wambrauw ${ }^{1}$, Sachiyo Osanai ${ }^{1}$, \\ Kazushige Honda', Satoshi Oku ${ }^{1,2}$, Hanako Shimura², Takashi Suzuki², Atsushi Yamasaki ${ }^{3}$, \\ Yosuke Okabe ${ }^{4}$, Keiji Ueno ${ }^{4}$ and Shuichi Onodera ${ }^{4}$
}

\author{
${ }^{1}$ Faculty of Agriculture and Life Science, Hirosaki University, Hirosaki 036-8561, Japan \\ ${ }^{2}$ Graduate School of Agriculture, Hokkaido University, Sapporo 060-0811, Japan \\ ${ }^{3}$ Japan National Agricultural Research Center for Tohoku Region, Morioka 960-2156, Japan \\ ${ }^{4}$ College of Agriculture, Food and Environment Sciences, Rakuno Gakuen University, Ebetsu 069-8501, Japan
}

Onions (Allium cepa L.) accumulate fructo-oligosaccharides (FOSs) as storage carbohydrates. FOSs exist in various chemical forms with different degrees of polymerization (DP) and chemical bonding states. Therefore, it is difficult to determine the precise FOS composition and quantity of each type of FOS. The objective of this study was to analyze the varietal differences in FOS composition and relative quantities among onion cultivars grown by spring-sown cultivation in Hirosaki City, Japan, using a high performance liquid chromatography (HPLC) system equipped with an evaporative light scattering detector (ELSD). FOS compositions of a number of varieties, as determined by HPLC/ELSD, were confirmed using matrix-assisted laser desorption/ionization time-of-flight -mass spectrometry (MALDI-TOF MS). Sugar and FOS analyses revealed distinct patterns of sugar and FOS accumulation, which led to categorization of the tested varieties into three types: (1) those with a relatively high overall quantity of FOSs and with a higher DP (DP3 to over DP9); (2) those containing mainly monosaccharides (fructose and glucose), disaccharide (sucrose), and low amounts of FOSs; and (3) those with a carbohydrate content that was intermediate compared to that of the other two types. Measurements of enzyme activities suggested that varietal differences in FOS composition are the result of differences in enzyme activities associated with the FOS biosynthetic and biodegradation pathways.

Key Words: fructan, HPLC, kestose, nystose, soluble carbohydrate.

\section{Introduction}

Onions (Allium cepa L.) are among the most common vegetables grown and consumed worldwide (Vågen and Slimestad, 2008). According to statistical data provided by the Ministry of Agriculture, Forestry

Received; November 14, 2016. Accepted; February 8, 2017.

First Published Online in J-STAGE on March 29, 2017.

This work was supported in part by a grant for Research and Development Projects for Application in Promoting New Policy of Agriculture, Forestry and Fisheries, from the Ministry of Agriculture, Forestry and Fisheries, Japan.

Parts of this study were presented at the 2013 Spring Meeting of the Japanese Society for Horticultural Science and the 29th International Horticultural Congress.

* Corresponding author (E-mail: t-maeda@hirosaki-u.ac.jp). and Fisheries of Japan, onions are primarily grown in Japan in two geographically isolated areas - the northernmost island, Hokkaido, and the southwestern region, including Saga and Hyogo - where they are produced by different cultivation methods. In Hokkaido, which has the coolest climate in Japan, long-day onion cultivars are sown in the spring. In contrast, in the warm southwestern Saga and Hyogo regions, relatively shortday onion cultivars are sown in the autumn. Onions are known to be rich sources of bioactive chemicals that are beneficial for human health including the so-called functional elements, such as flavonoids, sulfide compounds, and non-structural carbohydrates (Jaime et al., 2000; Tsushida and Suzuki, 1995; Vågen and Slimestad, 2008). Onions accumulate fructooligosaccharides (FOSs), a type of non-structural carbo- 
hydrate, as storage carbohydrates (Darbyshire and Henry, 1978, 1979; Shiomi et al., 2005). Sugars (mono and disaccharides, and FOSs) comprise up to $80 \%$ of the total dry weight of an onion bulb (Benkeblia et al., 2003; Kahane et al., 2001; Shiomi et al., 2005). It has been suggested that FOSs play a role in cold or draught stress tolerance in many plant species (Pilon-Smits et al., 1995; Pollock et al., 1988). In onions, it was proposed that FOSs contribute toward hardness and shelf life of the bulb (Suzuki and Cutcliffe, 1989).

The consumption of FOSs improves human health as they are a natural source of non-digestible dietary fiber (Bouhnik et al., 1999; Delzenne et al., 1995). FOSs have various chemical structures with different degrees of polymerization (DP) and chemical-bonding states in onion bulbs, and are categorized as either an inulin type or an inulin neo type. Therefore, determining the precise composition and quantity of each type of FOS is quite difficult. Onions have been reported to accumulate both types of FOSs (Darbyshire and Henry, 1978; Shiomi et al., 1991, 1997). A detailed FOS biosynthetic pathway, which includes the key metabolic enzymes and the chemical composition and structure of each type of FOS, has also been reported (Benkeblia et al., 2003, 2005; Fujishima et al., 2005; Shiomi et al., 2005). However, the differences in FOS composition and contents between common Japanese onion cultivars have not been comprehensively described. Varietal FOS differences could underlie the observed variations in onion bulb shelf life, environmental stress tolerance, and dietary effects on human health. Efforts to develop cultivars that have high FOS levels, which confer higher environmental stress tolerance (e.g., cold, drought), and have a notable functional effect on humans would be supported by a greater understanding of onion FOS composition.

High performance liquid chromatography (HPLC), which involves the use of a basic system with different columns and/or detectors, has been the main tool used for sugar and FOSs analysis in onions and many other plant species (Longland et al., 2012; Shiomi et al., 1991). A refractive index (RI) detector and a pulsed amperometric detector (PAD) are generally used for the identification of FOSs by HPLC. However, these methods have disadvantages such as sensitivity (RI detector) and machinery costs (PAD). Recently, an evaporative light scattering detector (ELSD) has been used to identify other non-UV-absorbent chemicals. A number of reports concerning determination of the FOS composition in onion bulbs using ELSD have been published (Downes and Terry, 2010; Vågen and Slimestad, 2008). These methods have a number of advantages, such as low machinery cost, simple and easy analysis procedures, and relatively high sensitivity, although they were not able to separate and quantitate the precise composition and content of each single FOS. The most significant advantage of these methods is the ability to easily obtain the FOS composition of each DP in the samples.

In this study, we employed a slightly modified method described by Downes and Terry (2010), which used a normal phase HPLC system equipped with an ELSD. Adequate peak separation in the sugar and FOS standards was observed and respectable calibration curves of fructose, glucose, sucrose, 1-kestose (DP3), and nystose (DP4) were obtained using this machinery (calibration curves of fructose and nystose are shown in Fig. 1A). In the current study, we present data resulting from the measurement of FOSs in 7 onion cultivars grown by spring-sown cultivation in Hirosaki City, Japan. Additionally, we measured enzymatic activities for FOS biosynthetic and biodegradation pathways of cultivars with different types of FOS accumulation.

\section{Materials and Methods}

\section{Plant materials}

Seven cultivars, namely-'Tokyo Red' (Open pollinated, Musashino Seed Co., Tokyo, Japan), 'Momiji No. 3' (F1, Shippo Co., Kagawa, Japan), 'Queru-Tama' (F1, Takii \& Co., Kyoto, Japan), 'Marusou' (F1, Kaneko Seed Co., Maebashi, Japan), 'Okhotsk 222' (F1, Shippo Co.), 'Pole Star' (Open pollinated, Watanabe Seed Co., Miyagi, Japan), and 'Kita-momiji 2000' (F1, Shippo Co.),-were used for this study. Of these cultivars, only 'Tokyo Red' displays red colored skin, while the others were yellow. 'Tokyo Red' and 'Momiji No. 3' are relatively early maturity types and are typically used for autumn-sowing cultivation in warm regions: however, our preliminary study revealed that these cultivars could be used for spring-sowing cultivation. 'Okhotsk 222' and 'Kita-momiji 2000' are long-day varieties, and are a popular choice in Hokkaido for use in spring-sowing cultivation. 'Pole Star', which is relatively less abundant in Japan (and mainly grown in the Tohoku region) is also used for spring-sowing cultivation. 'Queru-Tama' and 'Marusou' are varieties that have been recently released for spring-sowing cultivation, and according to seed company data, are early maturity types compared to the other varieties.

All seeds were sown in 448-hole cell trays filled with commercial nursery soil (Solid Soil; Minoru Sangyo Co., Okayama, Japan) on February 8, 2013. Sown trays were placed for 1 week in an air-conditioned room at $22^{\circ} \mathrm{C}$ to promote uniform germination and then transferred to a greenhouse with a heating system in the experimental field at Hirosaki University (Hirosaki City, Aomori, Japan, $\left.40.3^{\circ} \mathrm{N}, 140.3^{\circ} \mathrm{E}\right)$. The lower temperature limit inside the greenhouse was $5^{\circ} \mathrm{C}$. Seedlings were planted in an open field-(fertilized with nitrogen, phosphate, and potassium at concentrations of 1.5, 3.0, and $1.5 \mathrm{~kg} \cdot \mathrm{a}^{-1}$, respectively) - on April 22, by using a black plastic mulch with holes $(135 \mathrm{~cm}$ width, 4 rows, $25 \mathrm{~cm}$ apart $\times 12 \mathrm{~cm}$ between plants: Sankyo Co., 


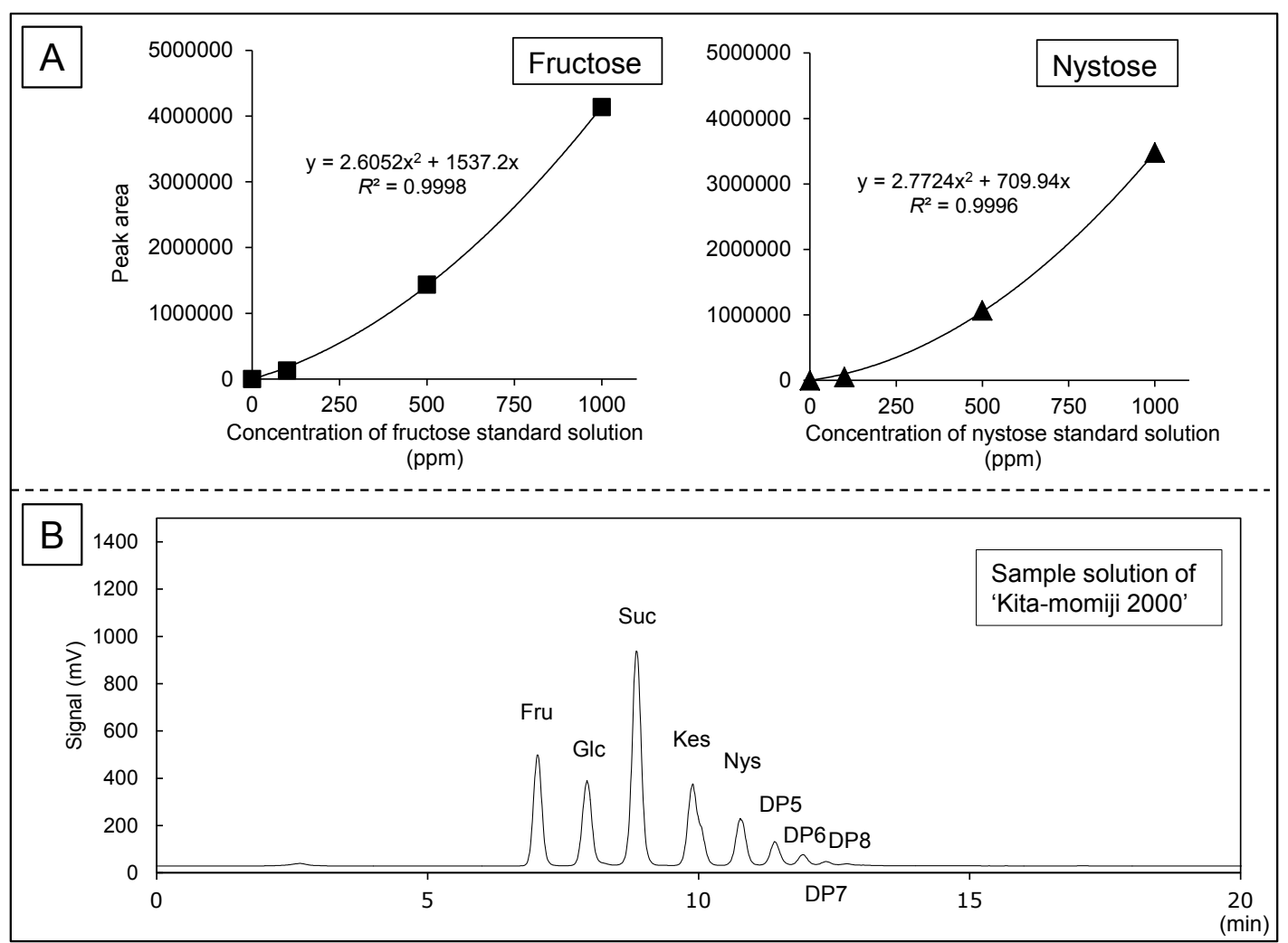

Fig. 1. (A): Calibration curves of Fru and Nys standard solutions. Standard solutions (0, 50, 500, and 1000 ppm) were analyzed using an HPLC system equipped with an ELSD. (B): A chromatogram of the sample solution ('Kita-momiji 2000').

Tokyo, Japan), to create a final planting density of 2222 plants $\cdot \mathrm{a}^{-1}$. Four replicates of 24 seedlings per cultivar were planted. Seedlings were cultivated under ambient precipitation with a number of pesticide sprays. The timing of plant lodging for each cultivar was monitored in order to decide the date of harvesting. The bulbs of each cultivar were harvested 3-8 days following plant lodging, after which the bulbs were naturally dried in a dark and cool room for approximately 2 weeks. The weight and size of each bulb was recorded, and the bulbs of replicate plantings were pooled. Then, 4-6 uniform-sized bulbs $(7.0-9.0 \mathrm{~cm}$ in diameter) of each variety were selected and frozen at $-18^{\circ} \mathrm{C}$ for subsequent sugar and FOSs analysis. Frozen bulbs were lyophilized using a FDU-1200 freeze drier (Tokyo Rikakikai Co., Tokyo, Japan) and milled to produce a fine powder using a TML-17 blender (Tescom Co., Tokyo, Japan).

\section{Extraction and quantification of sugar and FOSs}

Freeze-dried bulb powder $(20 \mathrm{mg})$ was placed into a $1.5 \mathrm{~mL}$ microtube and then heat treated at $95^{\circ} \mathrm{C}$ for $20 \mathrm{~min}$ in an electric water bath (HWA-50D; As One Co., Osaka, Japan) in order to inactivate enzymes involved in FOS degradation. Following this, sugars, including FOSs, were extracted in $1 \mathrm{~mL}$ of $95^{\circ} \mathrm{C}$ deionized water by incubating in a $95^{\circ} \mathrm{C}$ water bath for an additional $60 \mathrm{~min}$. Following this, samples were cen- trifuged at $4^{\circ} \mathrm{C}$ at a speed of $10000 \mathrm{rpm}$ (approximately $9000 \times g$ ), and $10 \mu \mathrm{L}$ of the supernatant was injected for the HPLC analysis. FOS content and composition in the sample solution were determined using a HPLC equipped with an ELSD and following a method described by Downes and Terry (2010) with slight modification. HPLC analysis was conducted using a Shimadzu LC10A VP system (Shimadzu Co., Kyoto, Japan) equipped with a Model 300S ELSD (M\&S Instruments Inc., Osaka, Japan) and Asahipak NH2P50-4E (4.6× $250 \mathrm{~mm}$ ) column (Showa Denko K.K., Kawasaki, Japan). The mobile phases consisted of acetonitrile (A) and deionized water (B). Analysis was performed by running each sample for $25 \mathrm{~min}$ at a column temperature of $40^{\circ} \mathrm{C}$ using a linear gradient at a flow rate of $1.0 \mathrm{~mL} \cdot \mathrm{min}^{-1}$. The gradients were as follows: $0 \mathrm{~min}$, $78 \%$ solvent $\mathrm{A}$ and $22 \%$ solvent $\mathrm{B} ; 25 \mathrm{~min}, 0 \%$ solvent $\mathrm{A}$ and $100 \%$ solvent B. An additional $15 \mathrm{~min}$ was needed as post running time in order to restore the solvent rates to the initial condition. The electrical signal from the ELSD was recorded using a personal computer and was analyzed using an HPLC data integrator system (Chromato-PRO; Run Time Instruments Co., Kanagawa, Japan). The chromatogram peaks for following a sugar standard solution (fructose [Fru] (Kanto chemical Co., Tokyo, Japan), glucose [Glc] (Kanto chemical Co.), sucrose [Suc] (Nacalai tesque Inc., Kyoto, Japan), 1-kestose [Kes] (Wako Pure Chemicals 
Ltd., Osaka, Japan), and nystose [Nys] (Wako Pure Chemicals Ltd.)) were confirmed by their injection in separate column runs. The content and composition of sugars and FOSs with lower DP (DP $\leq 4)$ in the sample solution were determined by calculating with calibration curves prepared using $0,50,500$, and $1000 \mathrm{ppm}$ of mixed external standard solution of Fru, Glc, Suc, Kes, and Nys. Meanwhile, the content of each FOS with higher DP ( DP $\geq 5)$ was calculated using the calibration curve of nystose and then expressed as a nystose equivalent value (mg Nys eq $\cdot \mathrm{g}^{-1}$ DW) because no commercial standard was available (Wang et al., 1999). Inulin type and inulin neo type were not separated with this quantification method. Therefore, one chromatogram peak represents the sum of isomers with different structures with same DP. Sample extractions and subsequent HPLC analyses were repeated in triplicate.

\section{FOS analysis using MALDI-TOF MS}

To identify the constituents of heterogeneous FOS contents in the onion samples, matrix assisted laser desorption/ionization time of flight mass spectrometry (MALDI-TOF MS) analysis was conducted. This analysis was conducted according to the methods of Suzuki et al. $(2011,2013)$ with slight modification. Ten milligrams of freeze-dried bulb powder was placed into a $1.5 \mathrm{~mL}$ microtube. The sample was extracted twice with $1.0 \mathrm{~mL}$ of $90^{\circ} \mathrm{C}$ deionized water for $30 \mathrm{~min}$ and then cooled slowly to room temperature. As an internal standard for FOS analysis, $10 \mu \mathrm{L}$ of a $4 \mathrm{mM} \gamma$ cyclodextrin was added to the first extracts. Following extraction, the cooled samples were centrifuged for $10 \mathrm{~min}$ at $13000 \mathrm{rpm}$ (approximately $12000 \times \mathrm{g}$ ). Each supernatant was transferred into a test tube and $1.0 \mathrm{~mL}$ of $100 \%(\mathrm{v} / \mathrm{v})$ ethanol was added. The mixture was concentrated to approximately $1.0 \mathrm{~mL}$ using a centrifuge evaporator (CVE-200D; Tokyo Rikakikai Co.) and these concentrated samples were used for MALDI-TOF MS analysis. 2,5-Dihydroxybenzoic acid (DHB) was used as a matrix for MALDI-TOF MS. Three microliters of each sample solution was placed onto a piece of Parafilm (Bemis Co., WI, USA) and mixed immediately with $3.0 \mu \mathrm{L}$ of matrix solution $\left(10 \mathrm{~g} \cdot \mathrm{L}^{-1} \mathrm{DHB}\right.$ in deionized water) by pipetting. Following this, $1.2 \mu \mathrm{L}$ of the mixture was mounted onto a sample slide for MALDI-TOF MS and air-dried in a desiccator. Analyses were performed using a MALDI-TOF MS instrument (Voyager-DE STR; Applied Biosystems, CA, USA) equipped with a nitrogen laser $(337 \mathrm{~nm})$, which produced 3-ns pulses at a repetition rate of $20 \mathrm{~Hz}$ with an accelerating voltage of $15 \mathrm{kV}$, and in the linear positive-ion mode with delayed extraction at $100 \mathrm{~ns}$. A manual control mode with laser intensity 2300 was used. All mass spectra were generated by collecting 50 laser shots that represented average masses. To compare the amounts of FOSs and $\gamma$-cyclodextrin, a minimum of independent spectra were obtained from the same target spot for normalization, and then the average value based on three target spots for each sample was used.

Measurement of enzyme activities in the biosynthesis and biodegradation pathways of FOSS

The bulbs of 'Kita-momiji 2000' and 'Pole Star' were used for analysis of enzyme activities. Harvested bulbs were air-dried and stored in a cool and dark room at Hirosaki University for approximately 2 months. Bulbs of a Uniform-size (7-9 cm in diameter) were sent to Rakuno Gakuen University and stored at $-40^{\circ} \mathrm{C}$ prior to analysis. Three bulbs for both 'Kita-momiji 2000' and 'Pole Star' were selected and a crude enzyme extract solution (CES) of each bulb was prepared as follows: $5 \mathrm{~g}$ of frozen bulb flesh was homogenized with $10 \mathrm{~mL}$ of $0.05 \mathrm{M}$ sodium phosphate buffer $(\mathrm{pH} 6.5)$ before it was filtered through cloth and centrifuged at $12500 \mathrm{rpm}$ (approximately $18800 \times \mathrm{g}$ ) for $35 \mathrm{~min}$ at $4^{\circ} \mathrm{C}$. The supernatant was collected, diluted to $20 \mathrm{~mL}$ with sodium phosphate buffer, and then concentrated to $1 \mathrm{~mL}$ by membrane ultrafiltration (Vivaspin 20; GE Healthcare Japan, Co., Tokyo, Japan). The total protein content in each CES was measured by the Bradford method (Bradford, 1976) using bovine serum albumin as a standard. The fructan: fructan 1fructosyltransferase (1-FFT, EC 2.4.1.100), fructan: fructan 6G-fructosyltransferase (6G-FFT, EC 2.4.1.243), and 1-kestose hydrolase enzyme activity assays were conducted as follows: $100 \mu \mathrm{L}$ of test solution consisting of $25 \mu \mathrm{L}$ McIlvaine buffer $\left(\mathrm{Na}_{2} \mathrm{HPO}_{4}\right.$-citric acid buffer $\mathrm{pH} 5.5$ ), $50 \mu \mathrm{L} 0.2 \mathrm{M}$ 1-kestose, and $25 \mu \mathrm{L}$ CES was incubated at $30^{\circ} \mathrm{C}$ and the enzymatic reaction was allowed to proceed for $1 \mathrm{~h}$, before it was stopped by heat treatment at $100^{\circ} \mathrm{C}$ for $3 \mathrm{~min}$. 1-FFT and 6GFFT activities were calculated based on the quantity of reaction products. The nystose hydrolase activity assay was conducted following a near-identical procedure to that described above, only with $0.2 \mathrm{M}$ nystose in place of $0.2 \mathrm{M} 1$-kestose solution in the test solution. The reaction products - nystose (DP4) for 1-FFT activity, neokestose (DP3) and $1^{\mathrm{F}}, 6^{\mathrm{G}}-\mathrm{di}-\beta_{-}{ }_{-}$-fructofuranosylsucrose (DP4) for 6G-FFT activity, and fructose for 1-kestose hydrolase activity - were determined using the high performance anion-exchange chromatography with pulsed amperometric detection (HPAEC-PAD) method described by Benkeblia et al. (2005). Nystose hydrolase activity was calculated based on the amount of fructose, as the reaction product. Each value of enzyme activity was expressed as Unit $\cdot \mathrm{mg}^{-1}$ protein (1 Unit is defined as that which catalyzes formation of $1 \mu \mathrm{mol}$ of reaction product in $1 \mathrm{~min}$ ).

\section{Results and Discussion}

Growth and yield

Results of the yield data are shown in Table 1. Bulbs of a typically commercial size were harvested in July with no significant cultivation problems for any of the 
Table 1. Date of lodging, date of harvest, average bulb weight, proportion of healthy bulbs of commercial size, and yields of seven cultivars grown in the field trial at Hirosaki University in 2013.

\begin{tabular}{|c|c|c|c|c|c|}
\hline Cultivar & Date of plant lodging & Date of harvest & $\begin{array}{l}\text { Ave. bulb weight } \\
\qquad(\mathrm{g})^{\mathrm{z}}\end{array}$ & $\begin{array}{l}\text { Rate of healthy, } \\
\text { commercial sized } \\
\text { bulbs }(\%)\end{array}$ & $\begin{array}{c}\text { Yields } \\
\left(\mathrm{kg} \cdot \mathrm{a}^{-1}\right)^{\mathrm{z}}\end{array}$ \\
\hline Momiji No. 3 & 11 Jul. & 16 Jul. & $150.1 \pm 17.4 \mathrm{~b}$ & 100.0 & $333.5 \pm 38.7$ \\
\hline Queru-Tama & $13 \mathrm{Jul}$. & $21 \mathrm{Jul}$. & $162.6 \pm 6.6 \mathrm{ab}$ & 96.8 & $349.8 \pm 14.2$ \\
\hline Marusou & 16 Jul. & 22 Jul. & $186.8 \pm 5.3 \mathrm{ab}$ & 93.8 & $389.4 \pm 11.1 \mathrm{a}$ \\
\hline Okhotsk 222 & 23 Jul. & $26 \mathrm{Jul}$. & $185.4 \pm 8.7 \mathrm{ab}$ & 96.4 & $397.1 \pm 18.6 \mathrm{a}$ \\
\hline Pole Star & 23 Jul. & 26 Jul. & $198.9 \pm 19.7 \mathrm{ab}$ & 83.3 & $368.1 \pm 36.6$ \\
\hline Kita-momiji 2000 & 26 Jul. & $31 \mathrm{Jul}$. & $206.9 \pm 4.2 \mathrm{a}$ & 95.8 & $440.4 \pm 9.0 a$ \\
\hline
\end{tabular}

${ }^{z}$ Means \pm SE. Different letters indicates significant differences (Tukey's HSD test $(P<0.05, \mathrm{n}=4)$ ).

tested varieties. 'Tokyo Red' showed the earliest maturation, and 'Kita-momiji 2000' was the last to mature among the seven tested varieties. Significant variation was observed in average bulb weight, which ranged from 150.1 ('Momiji No. 3') to $206.9 \mathrm{~g}$ ('Kita-momiji $\left.2000^{\prime}\right)$. Bulb weight appeared to gradually increase in accordance with the date of plant lodging; however, no significant difference was observed in terms of total yields. Each of the tested varieties displayed a yield from 332.9 to $440.4 \mathrm{~kg} \cdot \mathrm{a}^{-1}$, suggesting that all cultivars are suitable for spring-sowing cultivation in the Hirosaki district.

\section{Identification and confirmation of chromatogram peaks}

In the chromatogram produced using the carbohydrate sample solution of 'Kita-momiji 2000', peaks corresponding to Fru, Glc, Suc, Kes, and Nys were identified among other peaks (Fig. 1B). After hydrolyzing the sample $(0.5 \mathrm{~mL})$ in $1.5 \mathrm{~N} \mathrm{HCl}$ for $60 \mathrm{~min}$ at $90^{\circ} \mathrm{C}$, all peaks except those corresponding to Fru and Glc were degraded and the area of Fru and Glc peaks increased (data not shown). These results suggested that the compounds that resulted in peaks removed by $\mathrm{HCl}$ treatment contained polymers of Fru and Glc, consistent with FOS peaks. Comparison of our peak profiles with the former reports of Vågen and Slimestad (2008) and Downes and Terry (2010) indicated that FOSs derived from inulin and inulin-neo types would not likely be separated in our HPLC system. That is, one peak (of over DP $\geq 3$ ) consists of two different types of FOSs with the same DP. Clear chromatograms of FOSs were obtained from the sample solutions prepared from cultivars. The sugar and FOS composition of the 'Kitamomiji 2000' and 'Pole Star' samples were calculated using the calibration curves generated using standard solutions and are shown in Figure 2. Comparison of sugar and FOS composition between these two varieties indicated that they have different accumulation patterns of sugars and FOSs. In 'Kita-momiji 2000', Suc was the most abundant sugar while the variety contained a relatively higher level of DP FOSs. In contrast, 'Pole

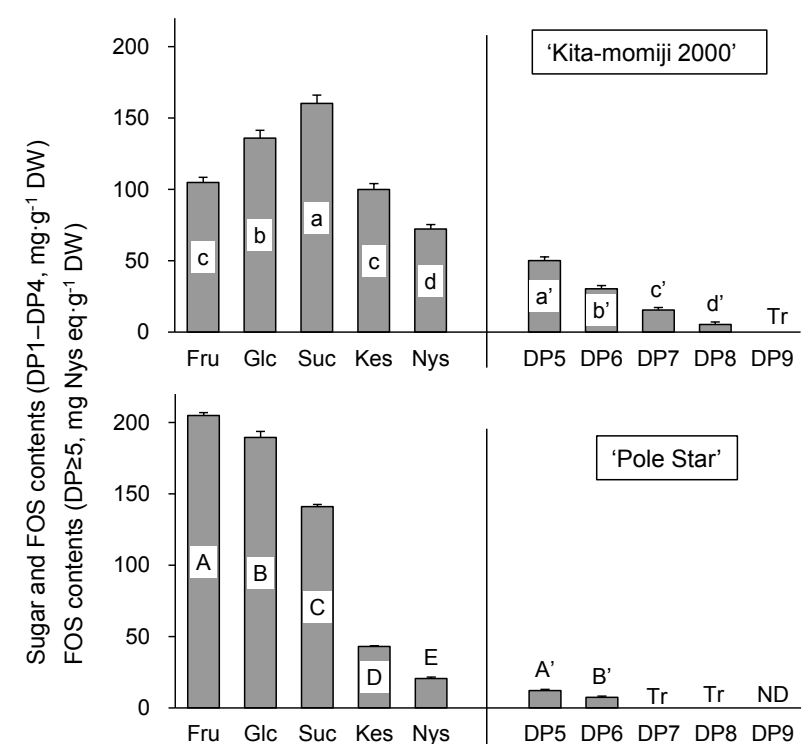

Fig. 2. Sugar and FOS composition in the bulbs of 'Kita-momiji 2000' and 'Pole Star'. Fru, Glc, Suc, Kes, and Nys were analyzed using the calibration curve of each sugar (mean $\pm \mathrm{SE}, \mathrm{n}$ $=3)$. FOS content of each DP $(\mathrm{DP} \geq 5)$ was expressed as nystose equivalent (mg Nys $\mathrm{eq}^{-\mathrm{g}^{-1}} \mathrm{DW}$ ) calculated using the calibration curve of Nys. Different letters indicate significant differences (Tukey's HSD test, $P<0.05, \mathrm{n}=3$ ). Owing to the differences in quantitation methods, Tukey's tests were conducted independently for the sugars and FOSs $(\mathrm{DP} \geq 5)$ of each variety. Tr: trace peak, ND: not detected.

Star' contained mainly mono and disaccharide (Frc, Glu, and Suc), and significantly lower FOSs contents.

In order to confirm these results, we next conducted MALDI-TOF MS analysis to determine the relative abundance of FOS types in these two varieties. Distinct peaks were observed in the MALDI-TOF MS analysis for the 'Kita-momiji 2000' sample (Fig. 3A). According to the reports of Suzuki et al. $(2011,2013)$, the $\mathrm{m} / \mathrm{z}$ value of each FOS is detected as the value of molecular mass plus that of the potassium ion $(\mathrm{m} / \mathrm{z}(\mathrm{DP}=\mathrm{n}$ FOS $)$ $\left.+\mathrm{m} / \mathrm{z}\left(\mathrm{K}^{+}\right)=180.2 \times \mathrm{n}-18.0 \times(\mathrm{n}-1)+39.1\right)$ because a FOS molecule is ionized with potassium under our MALDI-TOF MS conditions. The observed $\mathrm{m} / \mathrm{z}$ value 


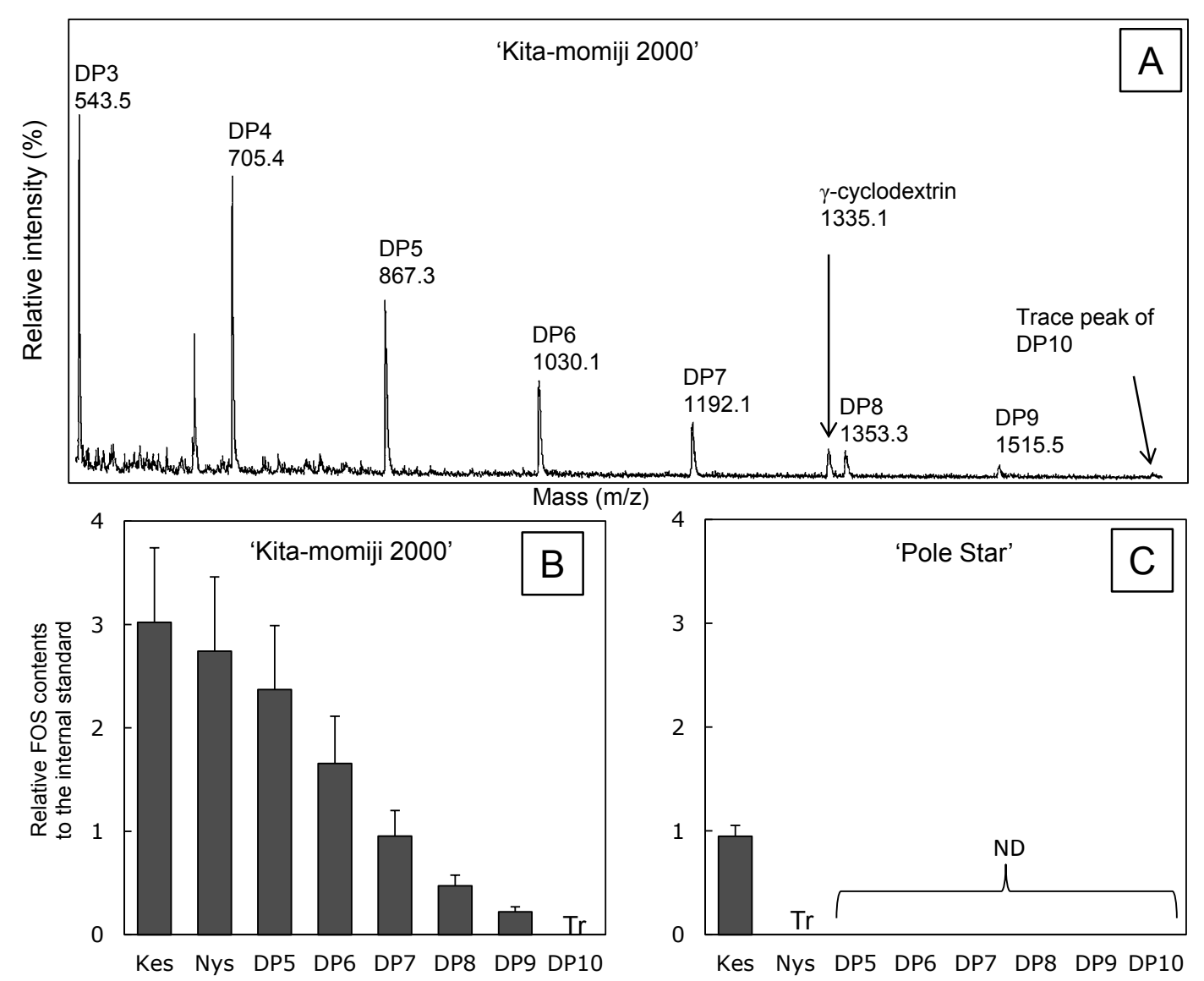

Fig. 3. Qualitative and semi quantitative analyses of FOSs using MALDI-TOF MS. (A): Mass spectrum of 'Kita-momiji 2000' sample solution. (B) and (C): Relative abundance of FOSs in 'Kita-momiji 2000' and 'Pole Star' sample solutions. Relative content of each FOS was calculated using the peak height of $\gamma$-cyclodextrin (internal standard; mean $\pm \mathrm{SD}, \mathrm{n}=9$ ). Tr: trace peak, ND: not detected.

of the peaks at $543.5,705.4,867.3,1030.1,1192.1$, and 1353.3 coincided well with those of the theoretical value of FOSs, with DP ranging from DP3 to DP8, respectively. In addition, a distinct peak of $\gamma$-cyclodextrin, included as an internal standard, was observed $(\mathrm{m} / \mathrm{z}$ $1335.1 \approx 162.2 \times 8+39.1=1335.9$; Fig. 3 A). We observed small peaks of FOS with DP9 and a trace peak of DP10. The relative abundance ratio of each FOS in the bulbs of 'Kita-momiji 2000' and 'Pole Star', calculated using the peak height of $\gamma$-cyclodextrin, is shown in Figure 3B and 3C. Wang et al. (1999) reported that DP3 to DP9 FOSs were detected in the aqueous extract from a red onion (the variety was not noted) using MALDI-TOF MS analysis. They also reported that the content of each DP tended to decrease in accordance with increases in DP. These results were similar to our own, suggesting that our analytical methods are applicable to this purpose. The ratio of each FOS type detected by MALDI-TOF MS analysis also agreed with the results from the HPLC analysis (Figs. 2, 3B, and 3C). This further supported that the peaks detected by the HPLC method employed in this study were those of FOSs.

A number of earlier reports suggested that MALDITOF MS is not suitable for certain quantitative analyses due to its poor repeatability as a result of the nonhomogeneous crystallization of the sample-matrix mixture (Gusev et al., 1995; Wang et al., 1999). In this study, a relatively wide variation was also observed between experimental MALDI-TOF MS runs, as shown in Figure 3B; however, individual experiments displayed adequate qualitative capacity and sensitivity, and these results yielded the same trends as in the former studies. As discussed above, the HPLC with ELSD method has disadvantages in terms of qualitative capacity due to a lack of selectivity in ELSD-based identification; however, the FOS content and composition determined by the method employed in this study correlated well with the relative abundance of FOS species analyzed by MALDI-TOF MS. Furthermore, once the identification of each peak is completed and the abundance ratio of FOSs is confirmed, the method employed in this study may become a simple, easy, and efficient approach for the quantitative analysis of FOSs in onion bulbs, without the need for additional confirmation by MS analysis.

Varietal differences in sugar and total FOS composition

Sugar and total FOS composition of the seven cultivars harvested in the field trial at Hirosaki University 
are shown in Table 2. Significant differences were observed between cultivars in the contents of each sugar and FOS (Tukey's HSD test, $P<0.05, \mathrm{n}=3$ ). In all the tested cultivars, the FOS content of a particular DP gradually decreased with increased levels of DP, regardless of total FOS contents (data not shown) as was observed in both 'Kita-momiji 2000' and 'Pole Star' shown in Figure 2. In the bulbs of 'Kita-momiji 2000', Suc was present at $160.3 \mathrm{mg} \cdot \mathrm{g}^{-1} \mathrm{DW}$, representing the most prevalent among those sugars and FOSs detected, followed by Glc $\left(136.1 \mathrm{mg} \cdot \mathrm{g}^{-1} \quad \mathrm{DW}\right)$ and Fru $\left(104.9 \mathrm{mg} \cdot \mathrm{g}^{-1} \mathrm{DW}\right)$. Total FOS content was $102.2 \mathrm{mg}$ Nys eq $\cdot \mathrm{g}^{-1} \mathrm{DW}$, which was the highest of the seven analyzed cultivars. Similar trends in sugar and FOS composition were observed in 'Queru-Tama' and 'Okhotsk 222'. The contents of FOSs in these varieties were 99.0 ('Queru-Tama') and $90.8 \mathrm{mg}$ Nys eq. $\mathrm{g}^{-1}$ ('Okhotsk $\left.222^{\prime}\right)$, respectively. In contrast, the monosaccharides Fru and Glc were present in high amounts, while relatively low Suc content and significantly low FOS amounts were detected in the bulbs of 'Pole Star' and 'Tokyo Red'. Monosaccharide contents of these varieties were significantly higher, and FOS contents were significantly lower, than other cultivars. We found 'Momiji NO. 3' and 'Marusou' contained a composition of sugars and FOSs composition intermediate to that of the 'Kita-momiji 2000' and 'Pole Star' types, which manifested as approximately equal amounts of Fru, Glc, and Suc and total FOS levels intermediate to those of 'Kita-momiji 2000' and 'Pole Star'.

As stated above, FOSs are important components of onions not only as a storage substance, but also contributing to culinary quality, tolerance against some environmental stresses, and even human health (Bouhnik et al., 1999; Delzenne et al., 1995; Pilon-Smits et al., 1995; Pollock et al., 1988; Suzuki and Cutcliffe, 1989). Thus, their content and composition are significant traits to be considered during the assessment of onion bulb quality. We next conducted a basic assessment of enzymatic activities in both the FOS biosynthetic and biodegradation pathways to further investigate the mechanism of varietal differences in FOS composition.

Enzyme activities in the biosynthesis and biodegradation pathways of FOSs

Previous studies reported that onions accumulate FOSs with DP9 to DP10 or more (Darbyshire and Henry, 1979; Downes and Terry, 2010; Shiomi et al., 2005; Vågen and Slimestad, 2008), while Vågen and Slimestad (2008) reported that some varieties mainly accumulated mono and disaccharides. However, few studies have focused on the contributing factors behind varietal differences in sugar and FOS composition. The results of our present study revealed that some onion varieties in Japan preferentially accumulate mono and disaccharides, which is similar to the results of Vagen and Slimestad (2008). Shiomi and his team have performed more than 10 years research on the FOS catabolic and anabolic pathways of plants, including onions (Benkeblia et al., 2003, 2005; Fujishima et al., 2005; Shiomi et al., 1991, 2005). According to their results, onions synthesize sucrose in their leaves from the monosaccharides produced by photosynthesis, and then sucrose is transferred to the young bulb. In the bulb, 1 -kestose (Kes, DP3) is synthesized from two sucrose molecules via a reaction catalyzed by the enzyme sucrose:sucrose 1-fructosyltransferase (SST), while nystose is synthesized from Kes, via a reaction catalyzed by the enzyme 1-FFT with a Kes molecule as a donor. During nystose biosynthesis, a Suc molecule is produced as a byproduct. In this manner, FOSs with single chain structure and higher DP (inulin type) are biosynthesized. Inulin type FOSs of any DP can be converted into inulin-neo type FOSs with a double chain structure, via a reaction catalyzed by the enzyme 6G-FFT, after which both Fru chains are further polymerized by the catalyst 1-FFT (Fig. 4A). Shiomi et al. (2005) also reported the following biodegradation pathway: the terminal chemical bond of the fructose chain of a FOS (DP $=\mathrm{n})$ molecule is hydrolyzed by the enzyme 1-FEH

Table 2. Sugar and FOS contents in onion bulbs harvested in the field trial at Hirosaki University.

\begin{tabular}{|c|c|c|c|c|c|c|}
\hline \multirow[b]{2}{*}{ Cultivar } & \multicolumn{5}{|c|}{ Sugar contents ${ }^{\mathrm{z}}$} & \multirow{2}{*}{$\begin{array}{l}\text { Total FOS content (Sum } \\
\text { of DP } \geq 5 \text { fructan })^{\mathrm{y}} \\
\left(\mathrm{mg} \mathrm{Nys} \mathrm{eq} \cdot \mathrm{g}^{-1} \mathrm{DW}\right)\end{array}$} \\
\hline & $\begin{array}{c}\text { Fructose } \\
\left(\mathrm{mg} \cdot \mathrm{g}^{-1} \mathrm{DW}\right)\end{array}$ & $\begin{array}{c}\text { Gulcose } \\
\left(\mathrm{mg} \cdot \mathrm{g}^{-1} \mathrm{DW}\right)\end{array}$ & $\begin{array}{c}\text { Sucrose } \\
\left(\mathrm{mg} \cdot \mathrm{g}^{-1} \mathrm{DW}\right)\end{array}$ & $\begin{array}{c}\text { Kestose } \\
\left(\mathrm{mg} \cdot \mathrm{g}^{-1} \mathrm{DW}\right)\end{array}$ & $\begin{array}{c}\text { Nystose } \\
\left(\mathrm{mg} \cdot \mathrm{g}^{-1} \mathrm{DW}\right)\end{array}$ & \\
\hline Tokyo Red & $203.0 \pm 3.0 \mathrm{a}^{\mathrm{x}}$ & $170.6 \pm 3.1 \mathrm{~b}$ & $142.7 \pm 5.3 \mathrm{~d}$ & $28.7 \pm 1.3 \mathrm{~d}$ & $11.6 \pm 0.2 \mathrm{~d}$ & $9.0 \pm 0.4 \mathrm{~d}$ \\
\hline Momiji No. 3 & $159.1 \pm 1.4 \mathrm{~b}$ & $152.2 \pm 3.0 \mathrm{c}$ & $147.9 \pm 2.2 \mathrm{~cd}$ & $80.2 \pm 2.2 \mathrm{~b}$ & $59.1 \pm 1.9 \mathrm{~b}$ & $81.4 \pm 4.7 \mathrm{~b}$ \\
\hline Queru-Tama & $133.6 \pm 1.4 \mathrm{c}$ & $141.4 \pm 3.8 \mathrm{~cd}$ & $178.1 \pm 2.1 \mathrm{a}$ & $99.0 \pm 1.4 \mathrm{a}$ & $67.8 \pm 1.3 \mathrm{a}$ & $99.0 \pm 2.2 \mathrm{a}$ \\
\hline Marusou & $157.1 \pm 3.3 b$ & $151.5 \pm 4.3 \mathrm{~cd}$ & $167.8 \pm 3.7 \mathrm{ab}$ & $85.3 \pm 2.4 \mathrm{~b}$ & $54.8 \pm 1.6 \mathrm{~b}$ & $76.1 \pm 2.6 \mathrm{~b}$ \\
\hline Okhotsk 222 & $128.3 \pm 1.8 \mathrm{c}$ & $143.9 \pm 2.4 \mathrm{~cd}$ & $178.4 \pm 0.3 \mathrm{a}$ & $103.4 \pm 0.3 \mathrm{a}$ & $69.4 \pm 0.3 \mathrm{a}$ & $90.8 \pm 1.6 \mathrm{a}$ \\
\hline Pole Star & $205.0 \pm 1.1 \mathrm{a}$ & $189.6 \pm 2.4 \mathrm{a}$ & $141.0 \pm 0.9 \mathrm{~d}$ & $43.0 \pm 0.3 \mathrm{c}$ & $20.5 \pm 0.7 \mathrm{c}$ & $22.7 \pm 2.6 \mathrm{c}$ \\
\hline Kita-momiji 2000 & $104.9 \pm 2.1 \mathrm{~d}$ & $136.1 \pm 3.1 \mathrm{~d}$ & $160.3 \pm 3.3 \mathrm{bc}$ & $100 \pm 2.3 \mathrm{a}$ & $72.2 \pm 1.8 \mathrm{a}$ & $102.2 \pm 5.7 \mathrm{a}$ \\
\hline
\end{tabular}

\footnotetext{
${ }^{z}$ Means \pm SE $(n=3)$.
}

y Total content of DP5 to DP9 fructan. The contents were caluculated using the nystose standard curve and expressed as equivalent values of nystose.

${ }^{x}$ Different letters indicates significant differences by Tukey's HSD test $(P<0.05, \mathrm{n}=3)$ conducted for varietal differences in the contents of each sugar or FOS. 


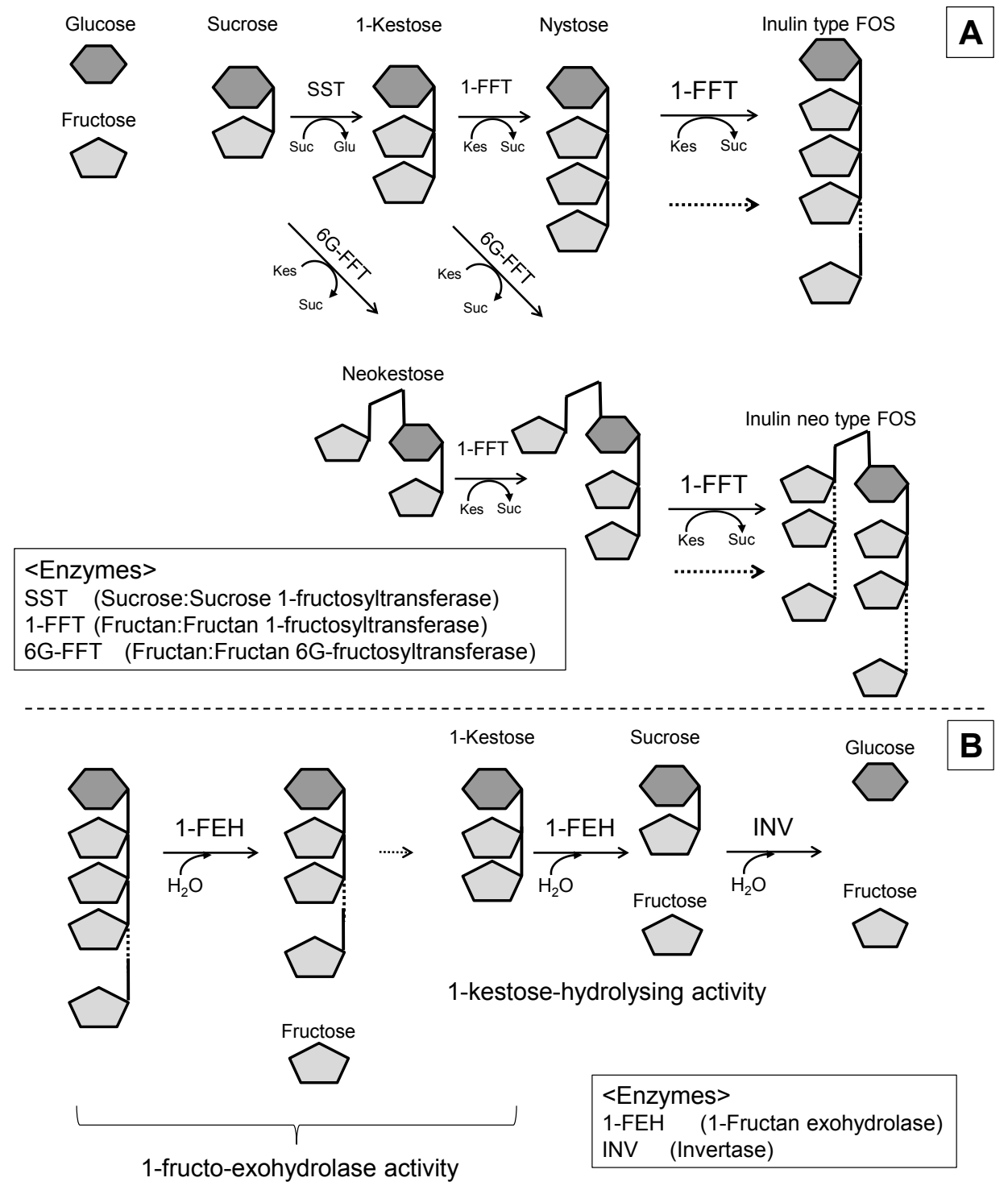

Fig. 4. Enzymatic biosynthesis (A) and biodegradation (B) pathways of FOSs in onion bulbs. The figure was drawn based on the reports of Fujishima et al. (2005) and Benkeblia et al. (2005).

(1-fructan exohydrolase). Then, the DP of the FOS chain is reduced one at a time (e.g., $\mathrm{DP}=\mathrm{n}-1, \mathrm{n}-2$, etc.), and finally, sucrose (DP2) is hydrolyzed into Fru and Glc by the enzyme INV (invertase) (Fig. 4B).

We conducted an assessment of the enzymatic activities involved in the onion FOS biosynthesis pathway (1SST, 1-FFT, and 6G-FFT) and of the activities involved in the biodegradation pathway (nystose hydrolase activity, 1-kestose hydrolase activity). These measurements were performed using two varieties with typical carbohydrate composition, namely 'Kita-momiji 2000' (accumulates FOSs with higher DP) and 'Pole Star' (accumulates mainly mono and disaccharides), both of which were grown and harvested during a field trial at Hirosaki University in 2013 (the sugar and FOS composition of each variety is shown in Fig. 2). Our measurements revealed that the tested biosynthetic enzymes (1FFT and 6G-FFT) showed activity in both 'Kita-momiji
2000' and 'Pole Star', and a significant difference in 6G-FFT activity was observed between the two varieties (Fig. 5A, B). In the biodegradation pathway, nystose hydrolase and 1-kestose hydrolase activities were detected in both cultivars (Fig. 5C, D). Due to a high degree of variation between replicate measurements, no significant difference was observed between the two cultivars for nystose hydrolase and 1-kestose hydrolase activity. The observation that 'Pole Star' - a variety that accumulates less FOSs - exhibited almost identical (or even stronger) enzyme activities in the biosynthetic pathway suggested that FOSs were at least produced in cultivar types such as 'Kita-momiji 2000' and 'Pole Star'. Suzuki and Cutcliffe (1989) reported that FOS contents were highest at harvest, and then decreased during storage while mono and disaccharides increased. Benkeblia et al. (2005) measured enzymatic activities involved in the FOS biodegradation pathway and re- 

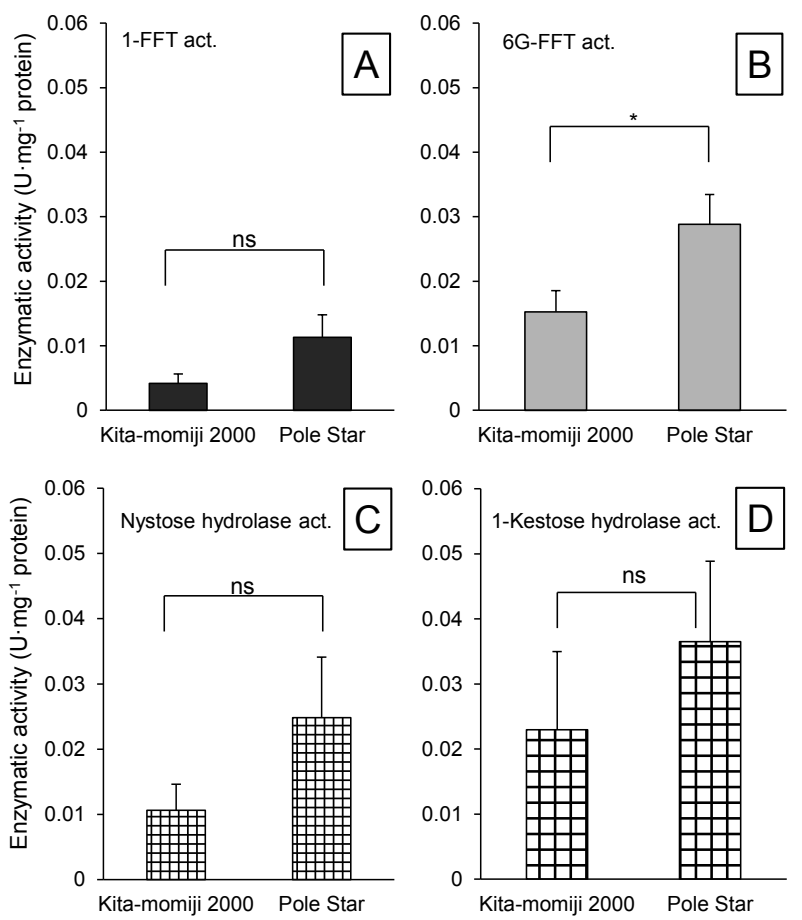

Fig. 5. Measurements of enzyme activity involved in FOS biosynthesis (A: 1-FFT, B: 6G-FFT) and biodegradation (C: Nystose hydrolase, D: 1-Kestose hydrolase) in onion bulbs. ns: not significant; *: significant difference ( $t$-test, $P<0.05, \mathrm{n}=3$ ).

ported that the degradative activities were changed during storage, leading to a change in sugar and FOS composition. Both of these studies were focused on cultivars containing relatively high amounts of FOSs with higher DP, and their results suggested that enzymes involved in both biosynthesis and biodegradation play important roles in determining sugar and FOS composition in onion bulbs. Thus, the results of the present study suggest that varietal differences in FOS composition may be caused by differences in balances of enzymatic activities in the FOS biosynthetic and biodegradation pathways. Because our enzymatic studies have just started, the varieties used and the measurement periods are quite limited. Therefore, we can only offer a limited interpretation. However, we predict that higher enzymatic rates within the FOS degradation pathway may naturally lead to decreased quantities of high-DP FOSs and the accumulation of mono and disaccharides. In addition, our measurements supported the hypotheses that, because Kes is an essential donor component in the FOS biosynthesis pathway, Kes degradation may contribute towards a decrease of FOS biosynthesis in addition to FOS contents. Further research is needed to elucidate the enzymatic activities in the FOS biosynthesis and biodegradation pathways, the differences among cultivars, and any changes in FOS levels from bulb formation until the end of onion storage.

In conclusion, we found that there are three different types of onion varieties concerning the composition of
FOSs: a type such as 'Kita-momiji 2000' that accumulates FOSs with relatively high DP, contains both inulin and inulin-neo type FOSs, and has the highest levels of Suc; a type such as 'Pole Star' that preferentially accumulates mono and disaccharides, and which may possess relatively higher enzymatic rates in the FOS biodegradation pathway; and a type that is intermediate between the first two types. Clarification of the mechanism and hereditary patterns of sugar and FOS biosynthesis, accumulation, and biodegradation may lead to the development of new varieties that have a higher tolerance for cold and/or drought stress or possess desirable dietary attributes. Likewise, a variety with a higher monosaccharide content may be sweeter and more palatable. Further study is still needed regarding the varietal differences in enzymatic activities, gene expression, and hereditary patterns for FOS biosynthesis in onions. The HPLC method we employed in this study could play an important role in determining the relative abundance and composition of sugars and the subtypes of FOS present in onion bulbs.

\section{Acknowledgements}

We thank all the seed companies for their generous supply of the test seeds.

\section{Literature Cited}

Benkeblia, N., S. Onodera and N. Shiomi. 2003. Effect of temperature and storage time on fructosyltransferase activities (1FFT and 6G-FFT) in onion bulb tissues. Acta Agric. Scand. 53: 211-214.

Benkeblia, N., S. Onodera and N. Shiomi. 2005. Variation of 1fructo-exohydrolase (1-FEH) and 1-kestose hydrolyzing (1$\mathrm{KH})$ activities, and fructo-oligosaccharides (FOS) status in onion bulbs. Influence of temperature and storage time. J. Sci. Food Agric. 85: 227-234.

Bouhnik, Y., K. Vahedi, L. Achour, A. Attar, J. Salfati, P. Pochart, P. Marteau, B. Flourié, F. Bornet and J. C. Rambaud. 1999. Short-chain fructo-oligosaccharide administration dosedependently increases fecal Bifidobacteria in healthy humans. J. Nutr. 129: 113-116.

Bradford, M. M. 1976. A rapid and sensitive method for quantitation of microgram quantities of protein utilizing the principle of protein-dye-binding. Anal. Biochem. 72: 248-254.

Darbyshire, B. and R. J. Henry. 1978. The distribution of fructans in onions. New Phytol. 81: 29-34.

Darbyshire, B. and R. J. Henry. 1979. The association of fructans with high percentage dry weight in onion cultivars suitable for dehydrating. J. Sci. Food Agric. 30: 1035-1038.

Delzenne, N., J. Aertssens, H. Verplaetse, M. Roccaro and M. Roberfroid. 1995. Effect of fermentable fructooligosaccharides on mineral, nitrogen and energy digestive balance in the rat. Life Sci. 57: 1579-1587.

Downes, K. and L. A. Terry. 2010. A new acetonitrile-free mobile phase method for LC-ELSD quantification of fructooligosaccharides in onion (Allium cepa L.). Talanta 82: 118124.

Fujishima, M., H. Sakai, K. Ueno, N. Takahashi, S. Onodera, N. Benkeblia and N. Shiomi. 2005. Purification and characterization of a fructosyltransferase from onion bulbs and its key role in the synthesis of fructo-oligosaccharides in vivo. New 
Phytol. 165: 513-524.

Gusev, A. I., W. R. Wilkinson, A. Proctor and D. M. Hercules. 1995. Improvement of signal reproducibility and matrix/ comatrix effects in MALDI analysis. Anal. Chem. 67: 10341041.

Jaime, L., F. Martínez, M. A. Martín-Cabrejas, E. Mollá, F. J. López-Andréu, K. W. Waldron and R. M. Esteban. 2000. Study of total fructan and fructooligosaccharides content in different onion tissues. J. Sci. Food Agric. 81: 177-182.

Kahane, R., E. Vialle-Guérin, I. Boukema, D. Tzanoudakis, C. Bellamy, C. Chamaux and C. Kik. 2001. Changes in nonstructural carbohydrate composition during bulbing in sweet and high-solid onions in field experiments. Environ. Exp. Bot. 45: 73-83.

Longland, A. C., M. S. Dhanoab and P. A. Harrisc. 2012. Comparison of a colorimetric and a high-performance liquid chromatography method for the determination of fructan in pasture grasses for horses. J. Sci. Food Agric. 92: 18781885.

Pilon-Smits, E. A. H., M. J. M. Ebskamp, M. J. Paul, M. J. W. Jeuken, P. J. Weisbeek and S. C. M. Smeekens. 1995. Improved performance of transgenic fructan-accumulating Tobacco under drought stress. Plant Physiol. 107: 125-130.

Pollock, C. J., C. F. Eagles and I. M. Sims. 1988. Effect of photoperiod and irradiance changes upon development of freezing tolerance and accumulation of soluble carbohydrate in seedlings of Lolium perenne grown at $2^{\circ} \mathrm{C}$. Ann. Bot. 62: $95-$ 100 .

Shiomi, N., N. Benkeblia and S. Onodera. 2005. The metabolism of the fructo-oligosaccharides in onion bulbs: A comprehen- sive review. J. Appl. Glycosci. 52: 121-127.

Shiomi, N., S. Onodera, N. J. Chatterton and P. A. Harrison. 1991. Separation of fructo-oligosaccharide isomers by anion-exchange chromatography. Agric. Biol. Chem. 55: $1427-1428$

Shiomi, N., S. Onodera and H. Sakai. 1997. Fructooligosaccharide content and fructosyltransferase activity during growth of onion bulbs. New Phytol. 136: 105-113.

Suzuki, M. and J. A. Cutcliffe. 1989. Fructans in onion in relation to storage life. Can. J. Plant Sci. 69: 1327-1333.

Suzuki, T., T. Maeda, S. Gramt, G. Grant and P. Sporns. 2013. Confirmation of fructans biosynthesized in vitro from [1-13C] glucose in asparagus tissues using MALDI-TOF MS and ESI-MS. J. Plant Physiol. 170: 715-722.

Suzuki, T., T. Maeda, S. Nomura, M. Suzuki, G. Grant and P. Sporns. 2011. Rapid analysis of fructans and comparison of fructan profiles in several different types of asparagus storage roots using MALDI-TOF MS. J. Hort. Biotech. 86: 210 216.

Tsushida, T. and M. Suzuki. 1995. Isolation of flavonoidglycosides in onion and identification by chemical synthesis of the glycosides. Nippon Shokuhin Kagaku Kaishi 42: 100 108.

Vågen, I. M. and R. Slimestad. 2008. Amount of characteristic compounds in 15 cultivars of onion (Allium cepa L.) in controlled field trials. J. Sci. Food Agric. 88: 404-411.

Wang, J., P. Sporns and N. H. Low. 1999. Analysis of food oligosaccharides using MALDI-MS: Quantification of fructooligosaccharides. J. Agric. Food Chem. 47: 1549-1557. 